\title{
Recent southern maser surveys
}

\author{
J. L. Caswell ${ }^{1}$ \\ ${ }^{1}$ Australia Telescope National Facility
}

\begin{abstract}
This discussion of recent maser surveys in the southern sky will allow us to see the context of the new methanol multibeam survey, which is currently in progress and described elsewhere at this symposium. My emphasis will be on masers that arise in star-forming regions in the disc of our Galaxy. I discuss outcomes from existing surveys, and comparison of different maser species, with special attention to the recognition of blue-shifted outflows.
\end{abstract}

Keywords. masers, surveys, stars: formation, ISM: molecules, radio lines: ISM

\section{Introduction}

A listing of 'Southern Galactic Plane surveys of $\mathrm{OH}$ and $\mathrm{H}_{2} \mathrm{O}$ Masers' (Caswell \& Haynes 1983) was given at a conference on 'Surveys of the Southern Galaxy' - paradoxically held at a rather northerly site - in the Netherlands! This meeting, in Australia, gives me the opportunity to redress the imbalance. Notably, 1983 was before the discovery of the widespread methanol masers at $6668 \mathrm{MHz}$. And it was before the completion of the Australia Telescope Compact Array, and thus positional accuracy of most known southern masers was poorer than 10 arcsec. Today I plan to bring the record up to date as to what is now available in 2007.

\subsection{The need for surveys}

Masers of $\mathrm{OH}$, methanol and water play three special roles in studying the birth of massive stars.

1. They are one of the earliest and most prominent indicators of the recent birth of a massive star, and they are a principal means of discovering such sites.

2. Each maser site is powered by a single embryonic massive star, but comprises many high brightness maser spots of small diameter and narrow velocity width, rendering them excellent kinematic probes.

3. Conditions necessary for maser excitation yield information on the chemical and physical conditions pertaining to the site at an early stage before the fully developed star has blown the evidence away.

I will cover three main topics:

firstly, I will present a brief summary of the more extensive surveys of the recent past, and their particular strengths; secondly, I will draw attention to some direct outcomes from the surveys, and additional outcomes that rely on comparisons of surveys; and finally I will discuss some recent surveys that have been conducted, but not yet published, and some preliminary results from them. 


\section{Recent surveys of methanol, $\mathrm{OH}$ and water}

\subsection{Methanol maser surveys}

For methanol, the Class II maser transition at 6668.5192 has received the most observing effort, and the compilation of survey data by Pestalozzi, Minier, Booth (2005) is an excellent starting point, in view of its extensive references. For that reason, I will not attempt to be exhaustive in my listing of references. However, it is important to stress that the original source material needs to be consulted, since it is very heterogeneous in its sensitivity, positional accuracy and general reliability; these limitations cannot be fully conveyed in any compilation and, furthermore, they lead to the occasional double listing of sources due to the apparently different positions in catalogues with poor positional accuracy.

I will distinguish between targeted surveys and 'blind' surveys. Menten's (1991) initial list of discoveries were the result of searches towards a variey of targets loosely regarded as tracers of star formation regions. The series of Hartebeesthoek surveys (starting with MacLeod, Gaylard, Nicholson (1992) and continuing to MacLeod, van der Walt, North et al. (1998)) primarily chose IRAS targets in the southern sky, and its detections usefully included some at Galactic latitudes too large to have been detected in typical blind surveys that are normally confined close to the Galactic plane; interestingly, it was also valuable in revealing the rather limited value of IRAS as a maser site predictor. From Torun, there was a similar search of IRAS sources in the northern sky (Szymczak, Hyrnek, Kus 2000). From Parkes there was a search of several hundred OH maser targets, with high success rate further demonstating the clear association of the two maser species, and providing the first extensive atlas of spectra with high sensitivity (Caswell, Vaile, Ellingsen et al. 1995a).

'Blind' surveys were conducted at Hobart with a $25 \mathrm{~m}$ dish looking in the southern sky from Galactic longitude 325 to 335 degrees (Ellingsen, von Bibra, McCulloch, et al. 1996b). This was later complemented by the Torun survey in the northern sky (Szymczak, Kus, Hrynek et al. 2002). More recently, Arecibo has been used in the small northern region accessible to it (Pandian, Goldsmith, Deshpande 2007), with the large collecting area providing a rapid surveying capability, combined with good sensitivity and fairly good positions.

In addition to the single dish surveys, we have an exploratory 'blind' survey from longitude 330 to 340 degrees made with the ATCA (Caswell 1996), followed by a similar survey of the Galactic Centre. ATCA follow-up to provide precise (sub-arcsecond) positional accuracy for masers discovered in single dish surveys has been made by Caswell, Vaile, Forster (1995b); Ellingsen, Norris, McCulloch (1996a); Caswell (1997); Phillips, Norris, Ellingsen, McCulloch (1998); Walsh, Burton, Hyland, Robinson (1998), and others.

\subsection{OH maser surveys}

In the southern sky, a series of Parkes surveys (culminating with Caswell \& Haynes 1987) made an extensive unbiassed 'blind' survey of 1665 and $1667-\mathrm{MHz}$ emission along the Galactic plane, and were followed by additional surveying, plus precise positioning of known masers, using the ATCA (Caswell 1998). The rarer $\mathrm{OH}$ transitions at 6035 and $6030 \mathrm{MHz}$ were surveyed at the locations of the main line (Caswell 2003), and likewise the 1720-MHz transition (Caswell 2004a). At $1612 \mathrm{MHz}$, a survey by Sevenster, Chapman, Habing et al. (1997a), Sevenster et al. (1997b), although optimised for late-type stars, is a valuable resource for recognising $1612-\mathrm{MHz}$ emission associated with SFR main line emission, and emphasises that in SFR regions the masers at this transition are quite rare, with comparable occurrence to the $1720-\mathrm{MHz}$ transition (see also Caswell 1999). 


\subsection{Water maser surveys}

'Blind' surveys for water masers have been extremely rare - essentially confined to a small pilot study done more than 20 years ago (Matthews, Olnon, Winnberg, Baud 1985), and a very recent survey of a small southern region (Breen et al. 2007), as shown in Shari Breen's poster. And yet large numbers of water masers are known, comparable to that of all of the other maser species. The water maser detections have accumulated over the past 30 years as a result of many very heterogeneous targeted surveys (e.g. Batchelor, Caswell, Goss et al. 1980). As noted later, there are new opportunities for investing effort into significant new unbiassed 'blind' surveys, and these promise to be very productive.

\section{Outcomes of the maser surveys}

\subsection{Direct outcomes}

Outcomes from even quite small targeted surveys can be very useful, but some classes of investigation require extensive 'blind' surveys.

1. From the only 'blind' survey covering an extensive portion of the Galactic plane, the series of $\mathrm{OH}$ surveys has yielded a Galactic distribution (Caswell \& Haynes 1987).

2. A luminosity function has also been derived for the $\mathrm{OH}$ masers, and is useful in estimating the productivity expected from future, deeper and more extensive surveys.

3. Estimations of a water maser luminosity function have been attempted, but mostly demonstrate the inadequate sensitivity and poor completeness of the input data.

4. Surveys of $\mathrm{OH}$ at 1720,6035 and $6030 \mathrm{MHz}$ have revealed the enormous potential of these transitions to map magnetic fields by utilizing the Zeeman effect; the effect is usually much easier to interpret in the case of these transitions, for which the splitting is inherently smaller, and propagation effects are far less prone to destroy one of the Zeeman components, than is the case for $1665-$ and $1667-\mathrm{MHz}$ masers.

5. Variability over many timescales is common amongst the majority of the maser species, and interpreted as a consequence of a variety of intrinsic and extrinsic effects. Some of the most striking variability occurs amongst the methanol masers, with remarkable outcomes as shown by Sharmila Goedhart at this meeting.

6. The typical maser velocity spread is another important parameter. To be valid, a study of this property is best confined to data from observations with good spatial resolution, so as to distinguish blends of nearby sources, and prevent the erroneous listing of wide velocity spreads that are merely blends.

7. Targeted surveys can provide a useful adjunct to pure 'blind' surveying. When targeted searches are conducted with a large beam, and are subsequently followed up with derivation of precise positions, masers that are offset from the targets are found, and these offset sources are rightly regarded as the outcome of an element of blind surveying.

8. A special case of the above occurs in the case of searches for Class I methanol masers at the location of known Class II methanol masers. Despite the known exclusivity of the two varieties of maser, such searches have been remarkably productive, with Class I masers generally offset from those of the Class II targets spatially (in most cases), and slightly offset in velocity. This conundrum of the relationship between Class I and Class II methanol masers is explored at this meeting by Simon Ellingsen. 


\subsection{Discoveries from comparisons of surveys}

After excluding the spuriously large apparent velocity spreads (arising from spatially nearby but distinct masers), there remain some $\mathrm{OH}$ and some methanol masers (and many water masers) with large velocity spreads. What then is the systemic velocity?

Among the methanol masers, there are relatively few with large widths (exceeding $12 \mathrm{~km} \mathrm{~s}^{-1}$ ) and most of these arise in sources where there is very strong emission near the median velocity and, extending from this, there are wings that are weak and roughly symmetrical. The systemic velocity then seems most likely to be at the median velocity. Overall, methanol seems to be an excellent indicator of systemic velocity.

In contrast to the methanol, there are some striking examples of $\mathrm{OH}$ masers with wide velocity spreads where the emission is concentrated at the velocity extremes. Where, in these cases, is the systemic velocity? Is it in the middle where there is no emission (as in the late-type stars where the front and back of an expanding shell dominate the emission)? Or is it at one extreme or the other; and if so, which extreme? And why?

To understand these $\mathrm{OH}$ masers, we can compare them with their methanol counterparts, with interesting results. Assuming that the methanol defines the systemic velocity, we find a number of $\mathrm{OH}$ masers showing outflows, with the most prominent ones being blue-shifted outflows. An example is $337.405-0.402$, first discovered by Caswell, Haynes \& Goss (1980), where features are clustered around velocity $-38 \mathrm{~km} \mathrm{~s}^{-1}$ (now known to be the same velocity as the methanol, and thus regarded as the systemic velocity) and a group separated in velocity (but not spatially) extending to at least $-56 \mathrm{~km} \mathrm{~s}^{-1}$. We interpret this as a blue-shifted outflow, and it is detected at $1667 \mathrm{MHz}$ as well as 1665 $\mathrm{MHz}$. Water maser emission is present but is confined to the systemic velocity range.

\section{Surveys in progress but not yet published}

With Chris Phillips, I have been conducting a water maser study at the locations of the southern SFR OH maser sites, using the ATCA. The use of the ATCA rather than a single dish has allowed immediate measurement of precise positions, and confirms that 170 of the $200 \mathrm{OH}$ masers searched have closely associated water masers; additional water masers found within the fields of view are quite common.

A 'blind' water maser search has been made, again with the ATCA, over two small (each 0.5 square degrees) regions of sky, chosen to straddle the Galactic plane, but to have only a few previously known masers. Preliminary results reveal more than 20 masers in the regions. Although these detections are not necessarily SFR masers, our initial expectation is that they are likely to be so, since, in a region close to the Galactic plane, SFR masers are likely to dominate in such a flux-limited sample.

Plans are in place to conduct a much more extensive water maser survey with the ATCA when the new correlator (the CABB) becomes operational in 2008. This will allow simultaneous coverage of a wide velocity range with no need to sacrifice spectral resolution, and continuum observations, recombination lines, and other spectral lines to be decided after preliminary tests.

Extensive water maser surveys are rightly regarded as the last frontier. They are difficult to conduct on account of the small beams at high frequency, and the discoveries are especially difficult to interpret without the benefit of pre-existing surveys of $\mathrm{OH}$ and methanol, since there may be significant numbers that are not of the SFR variety.

Interesting discoveries from the new water maser surveys so far include several that are DOMINATED by emission from a blue-shifted outflow. It will be interesting to monitor 
these to see whether any are of the exotic variety that show acceleration of the blue shifted outflow, as found for 291.284 - 0.716 and 291.270 - 0.719 (Caswell 2004b).

I conclude by mentioning one more 'survey' of $\mathrm{OH}$ masers - in this case, not searching for new masers, but conducting full spectropolarimetry on the full sample of known southern masers. This has been done with Chris Phillips using the Parkes telescope, with processing from the new spectral line processing package ASAP as described in a poster at this meeting by Chris Phillips \& Malte Marquarding. The resulting spectra from this survey reveal that linear polarization can be very prominent, despite the use of a single dish telescope (for which we might expect a tendency for polarization to be washed out when features are not spatially resolved). The atlas of these $\mathrm{OH}$ spectra therefore reveals which ones have especially interesting polarization, and provides an excellent way of determining priorities for observing at long baselines with the Australian Long Baseline Array (see Caswell \& Reynolds 2001, and poster papers at this meeting from Busaba Hutawarakorn Kramer and Indra Bains).

\section{Acknowledgements}

I thank my collaborators in the methanol multibeam (MMB) survey (co-authors of the paper by James Green in this symposium) for enthusiastic discussion of the role of maser surveys. I especially value the memory of time spent with Jim Cohen at Jodrell Bank, at Rose Cottage, and at Epping, Narrabri and Parkes, as we planned and began the MMB survey which, when completed, will be a lasting tribute to Jim.

\section{References}

Batchelor, R. A., Caswell, J. L., Goss, W. M., Haynes, R. F., Knowles, S. H., \& Wellington, K. J. 1980, AustJPhys 33, 139

Breen, S. L., Ellingsen, S. P., Johnston-Hollitt, M., Wotherspoon, S., Bains, I., Burton, M. G., Cunningham, M., Lo, N., Senkbeil, C. E., \& Wong, T. 2007, MNRAS 377, 491

Caswell, J. L. 1996, MNRAS 279, 79

Caswell, J. L. 1997, MNRAS 289, 203

Caswell, J. L. 1998, MNRAS 297, 215

Caswell, J. L. 1999, MNRAS 308, 683

Caswell, J. L. 2003, MNRAS 341, 551

Caswell, J. L. 2004a, MNRAS 349, 99

Caswell, J. L. 2004b, MNRAS 351, 279

Caswell, J. L., Haynes, R. F., \& Goss, W. M., 1980, AustJPhys 33, 639

Caswell, J. L., Haynes, R. F., 1983, in: W. B. Burton \& F. P. Israel (eds.), Surveys of the Southern Galaxy, Proceedings of a workshop, Leiden, Netherlands August 4-6 1982 (Dordrecht: D.Reidel Publishing) pp. 25-30

Caswell, J. L., Haynes, R. F., 1987, AustJPhys 40, 215

Caswell, J. L., Reynolds, J. E, 2001, MNRAS 325, 1346

Caswell, J. L., Vaile, R. A., Ellingsen, S. P., Whiteoak, J. B., \& Norris, R. P. 1995a, MNRAS 272,96

Caswell, J. L., Vaile, R. A., \& Forster, J. R. 1995b, MNRAS 277, 210

Ellingsen, S. P., Norris, R. P., \& McCulloch, P. M. 1996a MNRAS 279,101

Ellingsen, S. P., von Bibra, M. L., McCulloch, P. M., Norris, R. P., Deshpande, A. A., \& Phillips, C. J. $1996 \mathrm{~b} M N R A S 280,378$

MacLeod, G. C., Gaylard, M. J., \& Nicholson, G. D. 1992, MNRAS 254, 1P

MacLeod, G. C., van der Walt, D. J., North, A. J., Gaylard, M. J., Galt, J.A., \& MoriartySchieven, G. H. 1998, AJ 116, 2936

Matthews, H. E., Olnon, F. M., Winnberg, A., \& Baud, B. 1985, A\&\&A 149, 227

Menten, K. M. 1991, ApJ 380, L75

Pandian, J. D., Goldsmith, P. F., \& Deshpande, A. A. 2007, ApJ 656, 255 
Pestalozzi, M. R., Minier, V., \& Booth, R. S. 2005, A\& A 432, 737

Phillips, C. J., Norris, R. P., Ellingsen, S. P., \& McCulloch, P. M. 1998 MNRAS 300, 1131

Sevenster, M. N., Chapman, J. M., Habing, H. J., Killeen, N. E. B., \& Lindqvist, M. 1997a, $A \& A S 122,79$

Sevenster, M. N., Chapman,J. M., Habing, H. J., Killeen, N. E. B., \& Lindqvist, M. 1997b, $A \& A S 124,509$

Szymczak, M., Hyrnek, G., Kus, A. J. 2000, A\&AS 143, 269

Szymczak, M., Kus, A. J., Hrynek, G., Kepa, A., \& Pazderski, E. 2002, A\&A A 392, 277

Walsh, A. J, Burton, M. G., Hyland, A. R., \& Robinson, G. 1998, MNRAS 301, 640 